\title{
Comparison of acoustic rhinometry and nasal inspiratory peak flow as objective tools for nasal obstruction assessment in patients with chronic rhinosinusitis*
}

\author{
Efklidis K. Proimos', Devora E. Kiagiadaki', Theognosia S. Chimona', \\ Fokion G. Seferlis' ${ }^{1}$ Nicolas J. Maroudias², Chariton E. Papadakis' ${ }^{1}$ \\ ' ENT Department, Chania General Hospital, Chania, Crete, Greece \\ ENT Department, New Ionian General Hospital, Athens, Greece
}

Rhinology 53: 66-74, 2014

DOI:10.4193/Rhino13.203

*Received for publication:

November 23, 2013

Accepted: September 3, 2014

\begin{abstract}
Background: Chronic rhinosinusitis (CRS) is a disease with increasing incidence, characterized by persistent symptoms and negative impact on patient's quality of life. Nasal inspiratory peak flow (NIPF) and acoustic rhinometry (AR) were studied and compared as assessment tools for CRS with or without nasal polyps (NP), as well as, in follow up monitoring of conservative medical treatment.
\end{abstract}

Objectives: Seventy-eight patients were prospectively studied. Objective assessment included NIPF and AR at baseline and at 4 and 8 weeks after onset medical treatment. Measurements were studied in correlation with Sinonasal Outcome Test-22 (SNOT-22) questionnaire, Visual Analogue Scale (VAS) and endoscopic appearance score (EAS).

Results: Both NIPF and AR improved significantly, after medical treatment. NIPF score reflected CRS symptoms'improvement according to SNOT-22 total score at the end of treatment protocol, showing moderate to strong correlation with nasal obstruction VAS grading during the treatment period. AR measures were associated with EAS in all sets of examinations and correlated moderately with VAS scoring at 8 weeks.

Conclusion: NIPF and AR were proven to be promising methods for objective evaluation and monitoring of nasal obstruction based on different aspects of nasal physiology in patients with CRS.

Key words: chronic rhinosinusitis, peak nasal inspiratory flow, acoustic rhinometry, nasal obstruction

\section{Introduction}

According to the European Position Paper on Rhinosinusitis and Nasal Polyps (EPOS) $2012^{(1)}$, CRS with or without NP is defined as the presence of two or more symptoms, one of which should be either nasal blockage, obstruction, congestion, or nasal discharge (anterior/posterior nasal drip): with or without facial pain/pressure; with or without reduction or loss of smell, for more than 12 weeks. Symptomatology should be supported by demonstrable disease evident in either nasal endoscopy or Computed Tomography (CT) findings. Objective evaluation of patients with CRS has been challenging in daily practice, especi- ally when nasal congestion is the prominent symptom. Towards objective evaluation of the disease, numerous methods have been used, such as rhinomanometry (RM), NIPF and AR. In terms of anatomical aspects measurement of the nose, RM was developed in $1958^{(2)}$. It is a dynamic test of resistance to nasal airflow, measuring the pressure encountered by air passing through the nasal cavity ${ }^{(3)}$. It has been widely accepted as a valid functional method measuring nasal patency ${ }^{(4)}$ and evaluating the results of nasal surgery, however, its use in evaluation of medical treatment is not widely studied.

NIPF was described in 1980 by Youlten ${ }^{(5)}$. It is a dynamic mea- 
sure indicating the peak nasal airflow achieved during forced inspiration via a face mask, measured in liters per minute. The method is simple, easy to perform and is suggested to be reliable and reproducible. It is depended on the condition of the lower airway, measures both sides together and needs cooperation by the patient. Nasal inspiration correlates most with the subjective feeling of obstruction and is the best validated technique for monitoring nasal flow, in clinical trials and after nasal provocation ${ }^{(6)}$. Moreover, it was proven to correlate well with rhinomanometric measurements ${ }^{(7-11)}$.

AR was developed in 1991 as a means of evaluating nasal geometry by analyzing reflections of sound introduced into the nasal cavity ${ }^{(12)}$, and later was standardized by the Standardization Committee on Objective Assessment of the Nasal Airway of the European Rhinological Society ${ }^{(13)}$. It is an objective, reproducible, static measure of cross-sectional area as a function of distance into the nasal cavity, calculating volume over a specified depth. It does not depend on airflow and is less invasive and more expeditious than RM. It is considered a useful method for the assessment of rhinitis and structural abnormalities of the nasal airway regarding the outcome of medical treatment.

AR and NIPF have been found to correlate in a variable manner ${ }^{(7,14)}$ reflecting nasal pathology, mainly depending on patient's

Table1. Exclusion criteria.

1. Females who were pregnant or lactating.

2. Inability to follow the instructions within this protocol or known inability to attend all clinical visits within the intervals stated.

3. Patients under 18 years of age.

4. Systemic disease affecting the nose (cystic fibrosis, gross immunodeficiency congenital or acquired, congenital mucociliary problems e.g. primary ciliary dyskinesia).

5. Non-invasive fungal balls.

6. Systemic vasculitis and granulomatous diseases.

7. Patients who have any serious or unstable concurrent disease.

8. Any structural nasal abnormalities e.g. severe nasal septum deviation.

9. Medication affecting nasal mucosa.

10. Rhinosurgery during the past 6 weeks.

11. Use of systemic steroids 12 weeks before the study.

12. Medications for acute infections of the unified airway within 2 weeks before the first visit.

13. Patients with symptoms of Common Cold and post-viral rhinosinusitis.

14. Nasal polyps grade II-III. characteristics and treatment followed (surgical, conservative). Moreover, although their correlation with subjective sensation of nasal symptoms (e.g. nasal obstruction) has been proven (15), they have not been studied in relation with quality of life (QoL) instruments. In the present study, SNOT-22 was used, which is a 22-item questionnaire of CRS related symptoms, recently validated $^{(16)}$.

In patients with CRS, allergic rhinitis is a common coexisting disease showing increasing prevalence, however, the exact role of this finding it is not still clarified ${ }^{(17)}$. This lack of evidence contributed to our decision to evaluate patients on possible existence of allergic rhinitis.

The aim of our study was to investigate the possible role of objective measurements (AR and NIPF) for nasal obstruction assessment in CRS patients', in correlation with subjective measurements (rigid nasal endoscopy) and SNOT-22 test, before and after conservative treatment.

\section{Materials and methods}

\section{Patients}

This was a prospective study presented to 92 consecutive patients with CRS with or without NP, with nasal obstruction as the prominent symptom, assessed in the ENT Allergy and Endoscopy Clinic of our department. Exclusion criteria are shown in Table 1. From the 92 patients initially assessed, 6 were excluded after applying the exclusion criteria, 2 patients denied participation, 2 patients were lost in the 4 weeks follow-up and finally 4 were lost in the 8 weeks follow-up. The study was finally completed by 78 patients. The study protocol had been approved by Chania General Hospital Ethics Committee and informed consent was signed by all patients.

\section{Examination}

Patients' evaluation was performed at a baseline visit and at 4 and 8 weeks of medical treatment. All patients were examined in the same clinic with similar stable climate conditions, following always exactly the same order. Room temperature was $19^{\circ} \mathrm{C}$. All patients remained seated for 30 minutes to acclimatize before any measurement. Nasal endoscopy was first performed, which was followed by grading of the patients' symptoms with VAS and SNOT-22 test. This procedure lasted about 20-30 minutes for each patient and then NIPF was performed. Finally after an interval of at least 10 minutes, we proceeded with Acoustic Rhinometry. Decongestion was made with $0.1 \% \mathrm{w} / \mathrm{v}$ xylometazoline hydrochloride nasal solution, with one application per nostril ( $50 \mu \mathrm{g}$ of xylometazoline hydrochloride) three times each, and a 5-minute interval between them. Measurements with decongested nose were taken at least 15 minutes after nasal decongestion. All tests were performed in a sitting position. 
Medical treatment was applied according to EPOS 2012 guidelines for CRS with and without NP, based on symptoms severity (according to VAS). For patients with CRS without NP, medical treatment included nasal steroids and nasal douching. For nasal polyps group, medical treatment remained unchanged, without need of oral steroids, as only patients with polyp grade I that could be grouped together with CRS without NP were enrolled in the study.

All patients included in the study underwent diagnostic testing for allergic rhinitis with specific lgE tests.

\section{SNOT-22 test and VAS score}

All patients completed the SNOT-22 questionnaire before and at 4 and 8 weeks after onset of treatment. Symptoms were evaluated in the list: sleep, fatigue, productivity, cognitive function, emotional impact, cough, dizziness, facial pain and pressure, ear pain, nasal blockage, and sense of taste and smell. Each of 22 questions was scored with zero to five ( $0=$ indicating no problem, 5 = the worst possible symptom). The total score (0-110) was recorded in each set of investigations. The Minimal Clinically Important Difference (MCID), that is the smallest change in SNOT-22 score that can be detected by a patient, is 8.9 points ${ }^{(16)}$. The disease main symptom (nasal obstruction) was characterized as mild, moderate and severe at baseline visit, using the nasal symptom scoring criteria according to VAS score ${ }^{(18)}$. Patients were asked to indicate on a $10 \mathrm{~cm}$ VAS the level of severity of their symptoms and received treatment indicated for the grade of their disease. In VAS scale mild/moderate/severe are defined: Based on VAS scoring $(0-10 \mathrm{~cm})$ : mild as being $0-3$ inclusive, moderate as $>$ 3- 7 inclusive, and severe as $>7-10$ inclusive ${ }^{(19)}$.

\section{Rigid nasal endoscopy}

Rigid nasal endoscopy was performed in an organized manner with $0^{\circ} 4 \mathrm{~mm}$ rigid endoscope, with 3 passes. The first pass was along the floor of the nose to the nasopharynx permitting evaluation of the anatomy and of the condition of the nasal mucosa. The second pass was between middle and inferior turbinates permitting evaluation of anterior and inferior aspects of the middle meatus, fontanelles and accessory maxillary sinus ostia if any. In addition with passing the endoscope medial to the middle turbinate, it was visualized, if possible, the sphenoethmoidal recess, the superior turbinate and superior meatus. The third pass was performed rotating the endoscope under the middle turbinate offering posterior to anterior examination of bulla ethmoidalis, hiatus semilunaris, infundibulum, uncinate process, maxillary ostium, and frontal recess. Endoscopic appearance score (EAS), as described by Lund and Kennedy ${ }^{(18)}$, was recorded for each patient. Nasal polyps were rated from 0-3, and oedema, discharge and crusting from $0-2$, for both sides. Scoring ranged from 0 (minimum) to 18 (maximum). In all patients, nasal endoscopy was performed before decongestion.
NIPF

NIPF was performed with In-Check Inspiratory Flow Meter-HS Clement International, with the patient in upright sitting position. In all cases, before the examination, patients were given precise instructions. They were asked to inhale through the nose with a face mask firmly attached and with the mouth closed. The best of three trials was recorded (liters/minute). A flow less than $50 \mathrm{l} / \mathrm{min}$ was indicative of severe nasal obstruction, a flow between $50-80 \mathrm{l} / \mathrm{min}$ of moderate nasal obstruction, a flow between $80-120 \mathrm{l} / \mathrm{min}$ of mild, and finally a flow over $120 \mathrm{l} / \mathrm{min}$ was considered as normal ${ }^{(20)}$. A MCID of $20 \mathrm{l} / \mathrm{min}$ is recommended when NIPF is used as an outcome tool ${ }^{(21)}$.

\section{Acoustic rhinometry}

The method is based on the application of an audible sound impulse (click) of $150-10,000 \mathrm{~Hz}$ through a microphone and into the nostril of the subject. The signal is reflected in the opposite direction and the resultant information is digitized by a computer, displayed in a graph of the cross-section versus distance (or rhinogram). Three basic measurements are calculated: distance $(\mathrm{cm})$, minimum cross section area (MAC) $\left(\mathrm{cm}^{2}\right)$ and nasal cavity volume (NCV) $\left(\mathrm{cm}^{3}\right)$ at $0-3,3-5.2$ and $0-5 \mathrm{~cm}$ from the nose tip. Acoustic rhinometry was performed with an impulse acoustic rhinometer (RhinoMetrics, Rhinoscan Version 2.6 Edition $1.1^{\circ} 2003$, Interacoustics A/S, Assens) handled by two trained operators throughout the study. During the procedure, recommendations according to published protocols were followed, to establish accuracy in the recordings ${ }^{(22)}$. Measurements were taken 10 minutes after NIPF, before and 15 minutes after nasal decongestion with $0.1 \% \mathrm{w} / \mathrm{v}$ xylometazoline hydrochloride nasal solution, with one application per nostril $(50 \mu \mathrm{g}$ of xylometazoline hydrochloride) three times each, and a 5-minute interval between them. Acoustic rhinometry after decongestion was measured to determine more precisely whether the MCA corresponds to the nasal valve or the head of the inferior turbinate and was not compared to subjective symptoms. These measurements were not included in the study protocol.

Three recordings were made for each patient and the mean curve was calculated automatically for each nostril. According to the recommendation protocol ${ }^{(13)}$, nasal obstruction is mostly located in the anterior part of the nasal cavity, at a distance of $30 \mathrm{~mm}$ from the nares, represented by the first two notches of the rhinogram (I-notch and C-notch). The I-notch corresponds to MAC of the nasal vestibulum and valve area containing only limited congestive capacity. The C-notch corresponds to MAC of the turbinated region of the nasal cavity (head of the inferior turbinate), where the maximum congestive capacity of the nasal mucosa is located. After decongestion, we concluded that MAC corresponded to the $\mathrm{C}$-notch, located at a mean distance of 2.3 $( \pm 0.14) \mathrm{cm}$ from the nostril. NCV was chosen for nasal volume measurements $3-5 \mathrm{~cm}^{3}$ from the nares according to the recom- 
mendations, as being the most important measure for mucosal changes. For MAC, the sum value from right and left side was calculated, whereas for NCV mean value was used.

\section{Statistics}

Statistical analyses were performed with IBM ${ }^{\oplus}$ SPSS 20.0. Summary descriptive statistics are presented as mean standard deviation (SD) for continuous variables and as frequencies (\%) for categorical ones. Mean ( \pm SD) and percent (\%) differences of measurements between baseline and 4 and 8 weeks, as well as, total changes between baseline and the end of treatment protocol, were calculated.

Changes in scores before and after intervention were assessed with paired t-test or Wilcoxon test, as appropriate.

To assess associations between objective (MAC, NCV and NIPF) and subjective parameters (EAS and SNOT-22 test), Spearman correlations were computed. Independent t-test was performed to examine the ability of objective measurements to reflect patients' subjective response to therapy.

Pairs of measurements who exhibited significant correlations were further analyzed by means of linear regression equations to examine the extent of interaction of actual variance between them. This test can provide a more comprehensive possible clinical application of the correlations reported as it estimates the extent to which one or more explanatory variable can predict an outcome variable. F values (indicating strong association between the variables) and $\mathrm{R}$ values (multiple correlation coefficients) were calculated.

After applying the "cut-off" points for MCID in SNOT-22 total score and NIPF value provided by literature ${ }^{(16)}$, patients were further divided into "clinically important improvement" and "no
Table 2. Patients' characteristics.

$\begin{array}{cccc} & \text { Male }(\mathbf{n = 2 8}) & \text { Female }(\mathbf{n = 5 0 )} & \text { Total }(\mathbf{n = 7 8 )} \\ \begin{array}{c}\text { Age } \\ (\text { mean } \pm \text { SD) }\end{array} & 46 \pm 16 & 42.8 \pm 9.7 & 44 \pm 12.5 \\ \text { Allergy } & 22(78.6 \%) & 36(72 \%) & 50(74.4 \%) \\ \text { Nasal polyps } & 20(71.4 \%) & 20(40 \%) & 40(52.3 \%)\end{array}$

clinically important improvement" groups and were examined by means of cross-tabulation test. Moreover, patients were also grouped according to changes reported in VAS nasal obstruction score at all sets of examinations and the independent t-test was used to assess the relative distribution of variance between objective measurements. Effect sizes $\leq 0.2$ are considered weak, $>0.2$ and $\leq 0.5$ moderate, $>0.5$ and $\leq 0.8$ strong and $>0.8$ very strong.

Finally, to examine the possible negative effect of allergy on treatment outcome, the independent t-test was performed for changes of measurements between the groups of allergic and non-allergic patients.

All statistical tests were carried-out at the 2-sided $5 \%$ level of significance.

\section{Results}

Patients' characteristics are shown in Table 2. All measurements improved significantly 4 and 8 weeks after the baseline visit ( $p$ $<0.05$ ). Mean values for MAC and NCV, and median values for EAS, SNOT-22 total score, VAS nasal obstruction score and NIPF

Table 3. Values of measurements (mean, median) at baseline and 4 and 8 from the onset of treatment protocol and their relative changes expressed in $\%$.

\begin{tabular}{|c|c|c|c|c|c|c|}
\hline Measurements & Baseline & 4 weeks & 8 weeks & $\begin{array}{c}\text { Diff \% (baseline-4 } \\
\text { weeks) }\end{array}$ & $\begin{array}{l}\text { Diff } \%(4- \\
8 \text { weeks) }\end{array}$ & $\begin{array}{c}\text { Diff\%(baseline- } \\
\mathbf{8} \text { weeks) }\end{array}$ \\
\hline $\begin{array}{l}\text { EAS [median (min, } \\
\max ) \text { ] }\end{array}$ & $5(1,8)$ & $2(2,4)$ & $2(0,4)$ & $18 \%$ & $8 \%$ & $30 \%$ \\
\hline $\begin{array}{l}\text { SNOT-22 } \\
\text { [median(min, max)] }\end{array}$ & $34(16,94)$ & $13(8,34)$ & $10(0,59)$ & $56 \%$ & $9 \%$ & $64 \%$ \\
\hline $\begin{array}{l}\text { VAS nasal obstruc- } \\
\text { tion score [median } \\
(\min , \max )]\end{array}$ & $8(6,10)$ & $6(0,6)$ & $2(0,8)$ & $47 \%$ & $67 \%$ & $75 \%$ \\
\hline $\mathrm{MAC}[\operatorname{mean}(\mathrm{SD})]$ & $1(0.26)$ & $1.24(0.18)$ & $1.12(0.16)$ & $16 \%$ & $8 \%$ & $7 \%$ \\
\hline NCV [mean(SD)] & $3.9(1.5)$ & $5.3(1)$ & $4.8(0.8)$ & $-94.6 \%$ & $-95 \%$ & $41 \%$ \\
\hline $\begin{array}{l}\text { NIPF [median(min, } \\
\max )]\end{array}$ & $65(40,90)$ & $80(70,95)$ & $90(60,110)$ & $28 \%$ & $7 \%$ & $38 \%$ \\
\hline
\end{tabular}


Table 4. Summary results of simple linear regression analysis and correlation coefficients with levels of significance.

\begin{tabular}{|c|c|c|c|c|c|}
\hline Pair of measurements & rho (P) & $\mathbf{R}$ & $\mathbf{R} 2$ & $\mathbf{F}(\mathbf{P})$ & $t(P)$ \\
\hline NCVbaseline/EASbaseline & $-0.82(0.001)$ & 0.715 & 0.51 & $79.4(0.001)$ & $-8.916(0.001)$ \\
\hline NIPF8weeks/NCV8weeks & $0.61(0.001)$ & 0.71 & 0.49 & $75.03(0.001)$ & $-8.7(0.001)$ \\
\hline $\begin{array}{l}\text { NIPF8weeks/ } \\
\text { VAS nasal obstruction score8weeks }\end{array}$ & $-0.5(0.001)$ & 0.52 & 0.27 & $28.03(0.001)$ & $-5.3(0.001)$ \\
\hline $\begin{array}{l}\text { MAC8weeks/ } \\
\text { VAS nasal obstruction score8weeks }\end{array}$ & $-0.5(0.001)$ & 0.23 & 0.05 & $4.33(<0.05)$ & $-2.08(<0.05)$ \\
\hline MAC8weeks /EAS8weeks & $0.6(0.001)$ & 0.61 & 0.37 & $45.08(0.001)$ & $6.71(0.001)$ \\
\hline
\end{tabular}

Rho: Spearman correlation coefficient; R: multiple correlation coefficient; F: unexplained variation due to the regression/residual variation; t: unstandardized coefficient B/standard errors B.

for the 3 different time intervals are shown in Table 3, with their relative changes expressed in $\%$.

NIPF improved in a progressive manner, with an overall change of $38 \%$. Moreover, MAC and NCV values improved between baseline and 4 weeks, but decreased at 8 weeks; however, there was an overall improvement in scores for most of the patients by the end of treatment protocol ( $41 \%$ and $7 \%$, respectively). According to mean values of the VAS nasal obstruction score, patients reported severe nasal obstruction at baseline visit (median score 8), moderate symptoms at 4 weeks (median score 6), and mild symptoms at the end of treatment protocol (median score 2), showing the greatest overall change by $75 \%$. Similarly, SNOT22 total score improved in a progressive manner with overall change of $64 \%$, at 8 weeks. EAS was also improved progressively, being decreased by $30 \%$ at the end of the treatment protocol.

\section{Correlation and regression analysis}

\section{NIPF and AR parameters}

Significant moderate correlations were observed between NIPF values and NCV at baseline visit and at the end of treatment protocol ( $r$ o $=0.4$ and $r h o=0.6$, respectively, $\mathrm{p}<0.001$ ).

Correlations between objective and subjective measurements were present.

NIPF and EAS

No obvious correlations were observed.

\section{$A R$ and $E A S$}

A significant correlation was observed between MAC and EAS at baseline visit and at 8 weeks from the onset of treatment (rho $=0.4$ and 0.6 , respectively, $\mathrm{p}<0.001$ ). Moreover, NCV correlated significantly with EAS in all sets of investigations, notably at baseline visit (rho $=0.8,0.4$ and 0.2 , all $p<0.05$ ).
NIPF and SNOT-22 total score

NIPF showed a moderate correlation with the SNOT-22 total score at 8 weeks only ( $r h o=0.4, p<0.05$ ).

However, utilizing a cross-tabulation test based on MCID values for NIPF and SNOT-22 total score values between baseline visit and 4-weeks after onset of treatment, $76.9 \%$ of the patients reported clinically important differences in both measurements, whereas at the end of treatment protocol, this accounted for $81.3 \%$ of patients compared with the baseline visit and for $50 \%$ compared with values at 4 weeks.

AR and SNOT-22 total score

Total SNOT-22 score did not show any significant correlations with AR measurements.

The VAS nasal obstruction symptom

VAS nasal obstruction score correlated weakly with EAS and NIPF at baseline visit ( $r h o=0.2$ and 0.2 , respectively, $\mathrm{p}<0.05$ ). However, at the end of treatment protocol, it showed a moderate correlation with NIPF (rho $=0.5, p=0.001)$ and AR measures [with MAC (rho $=0.5, \mathrm{p}=0.001)$ and NCV $(\mathrm{rho}=0.3, \mathrm{p}<0.05)$ ]. Regression analysis

Applying regression analysis to pairs of measures with rho $\geq 0.5$, confirmed a strong association (high $F$ values and $R$ values greater than 0.5) for NCV/EAS at baseline visit, followed by NIPF/NCV and MAC/EAS and NIPF/VAS nasal obstruction scores at 8 weeks. At baseline visit, $51 \%$ of variation in NCV could be explained by EAS $\left(R^{2}=0.51\right)$. At the end of treatment protocol, $49 \%$ of variation in NIPF could be explained by NCV, $37 \%$ of variation in MAC by EAS and $27 \%$ of variation in VAS nasal obstruction score could be explained by NIPF.

Patients were further grouped according to change in grading 
Table 5. Summary of measurements by subjective evaluation scoring (VAS nasal obstruction score).

\begin{tabular}{|c|c|c|c|c|c|c|c|c|c|c|c|c|c|c|c|}
\hline \multirow[t]{2}{*}{$\begin{array}{l}\text { Diff/ } \\
\text { ces }\end{array}$} & \multicolumn{5}{|c|}{ 0-4 weeks } & \multicolumn{5}{|c|}{ 4-8 weeks } & \multicolumn{5}{|c|}{ 0-8 weeks } \\
\hline & $\begin{array}{c}\text { Group } \\
1 \\
\text { mean } \\
\text { (SD) }\end{array}$ & $\begin{array}{c}\text { Group } \\
2 \\
\text { mean } \\
\text { (SD) }\end{array}$ & $\begin{array}{l}\text { Effect } \\
\text { size } \\
\text { (SD) }\end{array}$ & $\begin{array}{l}\text { Mean } \\
\text { diffe- } \\
\text { rence, } \\
95 \% \mathrm{Cl}\end{array}$ & $\mathbf{P}$ & $\begin{array}{c}\text { Group } \\
1 \\
\text { mean } \\
\text { (SD) }\end{array}$ & $\begin{array}{c}\text { Group } \\
2 \\
\text { mean } \\
\text { (SD) }\end{array}$ & $\begin{array}{l}\text { Ef- } \\
\text { fect } \\
\text { size } \\
\text { (SD) }\end{array}$ & $\begin{array}{l}\text { Mean dif- } \\
\text { ference, } \\
95 \% \mathrm{Cl}\end{array}$ & $\mathbf{P}$ & $\begin{array}{c}\text { Group } \\
1 \\
\text { mean } \\
\text { (SD) }\end{array}$ & $\begin{array}{c}\text { Group } \\
2 \\
\text { mean } \\
\text { (SD) }\end{array}$ & $\begin{array}{l}\text { Effect } \\
\text { size } \\
\text { (SD) }\end{array}$ & $\begin{array}{l}\text { Mean dif- } \\
\text { ference, } \\
95 \% \mathrm{Cl}\end{array}$ & P \\
\hline NCV & $\begin{array}{c}1.9 \\
(0.3)\end{array}$ & $\begin{array}{c}1.3 \\
(1.5)\end{array}$ & 0.26 & $\begin{array}{c}0.6(-0.01 \\
1.3)\end{array}$ & 0.06 & $\begin{array}{l}-0.3 \\
(0.3)\end{array}$ & $\begin{array}{l}-0.5 \\
(1.0)\end{array}$ & 0.13 & $\begin{array}{c}0.2(-0.1 \\
0.6)\end{array}$ & 0.26 & $\begin{array}{l}-0.1 \\
(2.3)\end{array}$ & $\begin{array}{c}1.3 \\
(0.9)\end{array}$ & -0.37 & $\begin{array}{c}-1.4(-2.1 \\
-2.5)\end{array}$ & $<0.01$ \\
\hline EAS & $\begin{array}{c}3.9 \\
(1.4)\end{array}$ & $\begin{array}{c}1.7 \\
(2.3)\end{array}$ & 0.5 & $\begin{array}{c}2.1(1.03 \\
3.2)\end{array}$ & $<0.01$ & $\begin{array}{c}0.2 \\
(0.8)\end{array}$ & $\begin{array}{c}0.5 \\
(1.8)\end{array}$ & -0.1 & $\begin{array}{c}-0.3(-1 \\
0.4)\end{array}$ & 0.42 & $\begin{array}{l}-0.8 \\
(1.5)\end{array}$ & $\begin{array}{c}3.9 \\
(1.4)\end{array}$ & -0.85 & $\begin{array}{c}-4.7(-5.5 \\
,-4)\end{array}$ & $<0.01$ \\
\hline NIPF & $\begin{array}{l}20.5 \\
(9.0)\end{array}$ & $\begin{array}{c}11.9 \\
(12.3)\end{array}$ & 0.37 & $\begin{array}{c}8.6(2.5 \\
14.6)\end{array}$ & $<0.01$ & $\begin{array}{l}-6.5 \\
(4.1)\end{array}$ & $\begin{array}{l}11.3 \\
(8.3)\end{array}$ & -0.99 & $\begin{array}{c}-17.8(-21.3 \\
-14.4)\end{array}$ & $<0.01$ & $3.5(2.3)$ & $\begin{array}{c}25 \\
(17.2)\end{array}$ & -0.65 & $\begin{array}{c}-21.5(- \\
29.2,-13.7)\end{array}$ & $<0.01$ \\
\hline MAC & $\begin{array}{c}0.3 \\
(0.1)\end{array}$ & $\begin{array}{c}0.1 \\
(0.2)\end{array}$ & 0.53 & $\begin{array}{c}0.1(0.08 \\
0.2)\end{array}$ & $<0.01$ & $\begin{array}{l}-0.1 \\
(0.03)\end{array}$ & $\begin{array}{l}-0.1 \\
(0.2)\end{array}$ & 0.1 & $\begin{array}{c}-0.02(-0.1 \\
0.05)\end{array}$ & 0.53 & $\begin{array}{l}-0.12 \\
(0.3)\end{array}$ & $\begin{array}{l}0.16 \\
(0.2)\end{array}$ & -0.15 & $\begin{array}{c}-0.2(-4.1 \\
-0.1)\end{array}$ & $<0.01$ \\
\hline
\end{tabular}

$\mathrm{SD}=$ standard deviation; $\mathrm{Cl}=$ confidence interval; Effect size $=(($ mean $2-$ mean 1$)) /(\mathrm{SD}($ mean $2-$ mean 1$)) ;$ Diff/ces: changes of measurements between time intervals

of VAS nasal obstruction question, as appropriate in means of groups' size (distribution of VAS score), and independent t-testing was performed to estimate differences of variances of NIPF, EAS and AR measurements (Table 5). For the first time interval (baseline-4 weeks), patients were grouped in two groups, reported improvement of 2 (group 1 ) and $\geq 4$ points (group 2 ) in grading scale. From 4 to 8 weeks, patients reported worsening or no improvement were grouped separately (group 1) from those reported improvement (group 2). Finally, in terms of total change in score (baseline- 8 weeks), patients were grouped according to whether they had reported improvement of $<6$ (group 1) or $\geq 6$ points (group 2) in the grading scale.

Between baseline and 4 weeks, although the $p$-value was $<0.05$, no clinical measurement changed in concordance with subjective evaluation, as greater mean changes accounted for smaller changes in the VAS nasal obstruction score. In the second time interval, only NIPF could safely detect either subjective worsening or no improvement (decrease by mean of $6.54 \pm 4.1 \mathrm{l}$ / $\mathrm{min}$ ) and improvement (increase by mean of $11.35 \pm 8.36 \mathrm{l} / \mathrm{min}$ ). Considering total changes of measurements (baseline- 8 weeks), all measurements reflected patients' improvement, except for MAC which had a small effect size (0.15).

\section{Effect of allergy}

All measurements (subjective - objective) changed statistically in a different mode between allergic and non-allergic groups, between baseline and 4 weeks (Table 6). Changes in objective measurements were greater for the non-allergic group; however, allergic patients reported greater improvement in SNOT-22 as well in VAS nasal obstruction scores. Between 4 and 8 weeks, changes of AR measures as well as VAS nasal obstruction score were significantly different between allergic and non-allergic patients; in allergic patients, a decrease in MAC and NCV values was observed, as well as, a smaller improvement in VAS nasal obstruction score, compared with non-allergic patients. Finally, concerning the overall change of subjective and objective parameters, non-allergic patients showed a greater improvement only in EAS and MAC values, significantly different than allergic patients.

\section{Discussion}

In the present study, we evaluated the role of NIPF and AR as objective measurements for assessment and evaluation of treatment outcome, in patients with CRS with or without NP, at 4 and 8 weeks after onset of conservative treatment. In terms of correlations between them, significant correlation was observed between NIPF and NCV at the onset and at the end of treatment protocol. When studied in relation to subjective measurements, NIPF correlated significantly with the SNOT-22 total score and VAS nasal obstruction score, whereas AR parameters correlated significantly with EAS.

In recent literature, although both AR and NIPF have been studied in comparison with RM, few data are available for the correlation between the two methods. In a study of Numminen et al. ${ }^{(14)}$, where 69 adults patients with common cold were assessed 3 and 7 days from the onset of symptoms (no treatment administered), MCA and NCV showed statistical but not clinical significance with NIPF $(r<0.4, p<0.05)$. In the present study, NIPF was also moderately correlated with NCV before and after the treatment protocol. However, at the end of the treatment 
Table 6. Summary of measurements by allergy (allergic rhinitis).

\begin{tabular}{|c|c|c|c|c|c|c|c|c|c|c|c|c|c|c|c|}
\hline \multirow[t]{2}{*}{$\begin{array}{l}\text { Diff/ } \\
\text { ces }\end{array}$} & \multicolumn{5}{|c|}{ 0-4 weeks } & \multicolumn{6}{|c|}{ 4-8 weeks } & \multicolumn{4}{|c|}{ 0-8 weeks } \\
\hline & $\begin{array}{c}\text { Al- } \\
\text { lergic } \\
\text { group } \\
\text { [mean } \\
\text { (SD)] }\end{array}$ & $\begin{array}{c}\text { Non al- } \\
\text { lergic } \\
\text { group } \\
\text { [mean } \\
\text { (SD) }\end{array}$ & $\begin{array}{c}\text { Effect } \\
\text { size } \\
\text { (SD) }\end{array}$ & $\begin{array}{l}\text { Mean } \\
\text { diffe- } \\
\text { rence, } \\
95 \% \mathrm{Cl}\end{array}$ & $\mathbf{P}$ & $\begin{array}{c}\text { Al- } \\
\text { lergic } \\
\text { group } \\
\text { [mean } \\
\text { (SD)] }\end{array}$ & $\begin{array}{l}\text { Non al- } \\
\text { lergic } \\
\text { group } \\
\text { [mean } \\
\text { (SD) }\end{array}$ & $\begin{array}{l}\text { Ef- } \\
\text { fect } \\
\text { size } \\
\text { (SD) }\end{array}$ & $\begin{array}{l}\text { Mean dif- } \\
\text { ference, } \\
95 \% \mathrm{Cl}\end{array}$ & $\mathbf{P}$ & $\begin{array}{c}\text { Al- } \\
\text { lergic } \\
\text { group } \\
\text { [mean } \\
\text { (SD)] }\end{array}$ & $\begin{array}{c}\text { Non al- } \\
\text { lergic } \\
\text { group } \\
\text { [mean } \\
\text { (SD) }\end{array}$ & $\begin{array}{l}\text { Effect } \\
\text { size } \\
\text { (SD) }\end{array}$ & $\begin{array}{l}\text { Mean dif- } \\
\text { ference, } \\
95 \% \mathrm{Cl}\end{array}$ & $\mathbf{P}$ \\
\hline NCV & $\begin{array}{c}1.3 \\
(1.5)\end{array}$ & $\begin{array}{c}1.7 \\
(0.2)\end{array}$ & -0.2 & $\begin{array}{c}0.2 \\
(0.4,1.5)\end{array}$ & 0.001 & $\begin{array}{l}-0.7 \\
(0.8)\end{array}$ & $\begin{array}{l}0.07 \\
(0.7)\end{array}$ & -0.4 & $\begin{array}{c}0.2 \\
(0.3,1.2)\end{array}$ & 0.01 & $\begin{array}{c}0.6 \\
(1.7)\end{array}$ & $\begin{array}{c}1.7 \\
(0.7)\end{array}$ & -0.3 & $\begin{array}{c}0.2 \\
(-0.07,0.7)\end{array}$ & 0.1 \\
\hline EAS & $\begin{array}{c}2.0 \\
(2.6)\end{array}$ & $\begin{array}{c}3.0 \\
(0.0)\end{array}$ & -0.3 & $\begin{array}{c}0.3 \\
(0.5,2)\end{array}$ & 0.02 & $\begin{array}{c}0.3 \\
(1.8)\end{array}$ & $\begin{array}{c}0.7 \\
(0.5)\end{array}$ & -0.14 & $\begin{array}{c}0.2 \\
(-0.1,0.8)\end{array}$ & 0.1 & $\begin{array}{c}2.4 \\
(2.8)\end{array}$ & $\begin{array}{c}3.7 \\
(0.5)\end{array}$ & -0.3 & $\begin{array}{c}0.3 \\
(0.2,1.6)\end{array}$ & 0.01 \\
\hline NIPF & $\begin{array}{l}11.9 \\
(12)\end{array}$ & $\begin{array}{c}20.5 \\
(0.01)\end{array}$ & -0.4 & $\begin{array}{c}2.5 \\
(3.3,13.8)\end{array}$ & 0.01 & $\begin{array}{c}5.8 \\
(11.9)\end{array}$ & $4(8.5)$ & 0.08 & $\begin{array}{c}2.8 \\
(-7.6,3.9)\end{array}$ & 0.5 & $\begin{array}{c}17.7 \\
(17.5)\end{array}$ & $\begin{array}{c}24.5 \\
(17.3)\end{array}$ & -0.19 & $\begin{array}{c}4.5 \\
(-2.4,15.9)\end{array}$ & 0.1 \\
\hline MAC & $\begin{array}{c}0.1 \\
(0.2)\end{array}$ & $\begin{array}{c}0.3 \\
(0.1)\end{array}$ & 0.5 & $\begin{array}{c}0.04 \\
(0.2,0.4)\end{array}$ & 0.001 & $\begin{array}{l}-0.1 \\
(0.2)\end{array}$ & $\begin{array}{l}0.008 \\
(0.04)\end{array}$ & -0.3 & $\begin{array}{c}0.02 \\
(0.1,0.2)\end{array}$ & 0.01 & $\begin{array}{l}0.01 \\
(0.2)\end{array}$ & $\begin{array}{l}0.32 \\
(0.1)\end{array}$ & -0.7 & $\begin{array}{c}0.04 \\
(0.06,0.2)\end{array}$ & 0.01 \\
\hline $\begin{array}{l}\text { SNOT } \\
-22\end{array}$ & $\begin{array}{c}-28.6 \\
(15)\end{array}$ & $\begin{array}{r}-12.2 \\
(5.1)\end{array}$ & -0.5 & $\begin{array}{c}16.4 \\
(9.3,23.5)\end{array}$ & 0.001 & $\begin{array}{c}-0.3 \\
(17.1)\end{array}$ & $\begin{array}{l}-7.5 \\
(1.3)\end{array}$ & 0.3 & $\begin{array}{c}-7.4 \\
(-15.1 \\
0.22)\end{array}$ & 0.5 & $\begin{array}{c}28.6 \\
(24.1)\end{array}$ & $\begin{array}{l}19.7 \\
(3.9)\end{array}$ & 0.2 & $\begin{array}{c}-8.9 \\
(-19.7,1.8)\end{array}$ & 0.1 \\
\hline $\begin{array}{l}\text { VAS } \\
\text { nasal } \\
\text { ob- } \\
\text { struc- } \\
\text { tion } \\
\text { score }\end{array}$ & $\begin{array}{c}-4.0 \\
(0.94)\end{array}$ & $\begin{array}{c}-2.6 \\
(0.91)\end{array}$ & -0.7 & $\begin{array}{c}1.4 \\
(0.9,1.8)\end{array}$ & 0.001 & $\begin{array}{l}-1.7 \\
(2.3)\end{array}$ & $\begin{array}{c}-3.4 \\
(0.94)\end{array}$ & -0.4 & $\begin{array}{c}-1.6 \\
(-2.4,0.9) \\
-1.6\end{array}$ & 0.01 & $\begin{array}{c}5.7 \\
(1.9)\end{array}$ & $\begin{array}{c}6.0 \\
(0.0)\end{array}$ & -0.15 & $\begin{array}{c}0.27 \\
(-0.7,0.2)\end{array}$ & 0.3 \\
\hline
\end{tabular}

$\mathrm{SD}=$ standard deviation; $\mathrm{Cl}=$ confidence interval; Effect size $=($ (mean non allergic - mean allergic $)) /(\mathrm{SD}$ (mean non allergic $-\mathrm{mean}$ allergic $)) ;$ Diff/ces: changes of measurements between time intervals.

protocol, NCV could predict $49 \%$ of variance in NIPF $\left(R^{2}=0.49\right)$. In clinical practice, this can be considered an interesting observation for the comparison of two methods of different nature (static vs. dynamic) and could suggest the complementary use of both of them assessing different aspects of nasal pathology. According to EPOS 2012, NIPF is considered a simple and quick method of measuring nasal airflow and although its role is limited in CRS assessment, it can be useful in patients with moderate to severe nasal obstruction, as an objective marker of response to medical treatment. Its correlation with subjective evaluation of nasal patency has been reported in healthy subjects ${ }^{(23,24)}$, as well as, in patients who underwent nasal surgery (11). In the present study, NIPF showed a significant but weak correlation with SNOT-22 total score, especially at baseline visit (rho $=0.2$ ). VAS scoring of "nasal obstruction symptom" before and at the end of the treatment protocol correlated more effectively with NIPF, with a clinically significant association at the end of the treatment protocol ( $r h o=0.61$ ), as confirmed from regression analysis. Moreover, considering MCID values for NIPF and SNOT-22, NIPF was proven to predict patients' evaluation in the majority of them, especially in the beginning and at the end of treatment protocol. However, a categorical scale of self-grading nasal obstruction (such as VAS score) corresponded more accurately with air flow rates than SNOT-22 total score, enhancing its role in clinical practice. Furthermore, NIPF has proven a sensitive marker of worsening or non-improvement of nasal obstruction symptoms, as seen in the patient group 1 between 4 and 8 weeks of the treatment protocol, as well as of the overall grade of improvement, where increase of NIPF by $25 \mathrm{l} / \mathrm{min}$ corresponded with improvement of $\geq 3$ points in the grading scale.

AR has been used in numerous studies; however, only in a few has it been compared with subjective evaluation methods. A correlation with subjective symptoms was reported before and after surgical intervention in the majority of them ${ }^{(25-28)}$, and when examined on a group level, AR measures correlated significantly with VAS or subjective patient evaluation regarding nasal obstruction ${ }^{(29)}$. Moreover, according to a study of Larson et al., a poor correlation between AR and subjective nasal obstruction scores was reported in healthy individuals, in contrast with congested subjects ${ }^{(30)}$. However, no data are available for the correlation of AR with subjective measurements for patients with CRS before and after conservative treatment, where AR is compared to validated QoL instruments, such as SNOT-22. In our 
study, AR parameters correlated significantly only with VAS scoring of nasal obstruction symptoms at the end of the treatment protocol. However, in the baseline visit, when only patients, who graded this question $>6$ were included, scoring of nasal obstruction symptoms correlated significantly with NCV and MAC (rho $=0.43$ and 0.56 , respectively, $p=0.001$ ). This indicates that AR could provide partially an objective confirmation of nasal obstruction symptoms in patients with normal-mild (score $0-1$ ) as well as with severe (score 7-10) disease.

AR has been studied in accordance with nasal cavity dimensions measured by $C T$, showing significant correlations, especially in the anterior nasal cavity, including notches I and C $(p=0.001)$ (31). Moreover, in a study of Gorey et al., AR proved to correspond to nasal anatomical landmarks measured with rigid nasal endoscopy, especially in the nasal valve and anterior end of inferior turbinate ${ }^{(32)}$. These observations may explain the association between AR parameters and EAS, although rigid nasal endoscopy could be characterized as a subjectively interpreted rhinometric method. Moreover, one could expect AR to correlate with the endoscopic appearance of the nose as it reflects nasal anatomy in two (MAC) and three (NCV) dimensions, respectively. In the present study, EAS was used, which is a clinically validated grading scale and significant correlations were found with MAC and NCV before and at the end of the treatment, which was confirmed by regression analysis.

A recent review study highlighted the issue of validated questionnaires use in clinical practice and for research purposes, to extract generalized results comparing different studies ${ }^{(33)}$. As far as subjective evaluation of CRS symptoms and more specifically of nasal obstruction is concerned, we used the validated SNOT22 QoL questionnaire and studied the association of total scoring as well as VAS scoring of nasal obstruction symptoms, with objective measurements. Objective measurements seemed to confirm the patients' evaluation in the majority of cases, mainly at baseline and the end of treatment protocol (SNOT-22 total score), with correlations to be greater and more accurate when just the VAS score was examined. However, in our study, correlations were higher after treatment was completed, indicating that objective measurements could confirm subjective improvement after conservative treatment.

Allergy had a high prevalence in our group of patients, being present in $74.5 \%$ of them. SNOT-22 score was more impaired in the allergy group (mean value 44 vs 33 ) at baseline visit whereas nasal obstruction was VAS scored as severe (median value 8 ) in both groups. Our results are in accordance with a multicenter, observational, cross-sectional study of Davila et al. according to which, atopy worsened CRS patients QoL ${ }^{(34)}$. Moreover, allergic patients reported greater improvement in SNOT-22 as well as in VAS nasal obstruction score after medical treatment. These findings were not documented with objective measurements, which showed a greater change for the non-allergic group. Acoustic rhinometry was previously mentioned to change along with subjective feeling of obstruction during treatment, in patients with allergic rhinitis ${ }^{(35)}$. In our study, allergy had an inverse effect on objective measurements and their change during the treatment period. The response to medical treatment differed constantly between allergic and non-allergic patients, as far as AR measures are concerned; their improvement was less profound in allergic patients, and their values worsened between 4 and 8 weeks. However, in our study, AR measures didn't change always along with nasal obstruction symptoms and the overall improvement was not different in comparison with non-allergic patients.

As far as potential parameters not considered in our study, there was no further analysis performed to trace the influence of parameters as gender, Body Mass Index (BMI) and smoking, which are previously reported to affect AR measurements, as our sample size could not support a multifactorial statistical analysis.

In conclusion, in the present study, we attempted to examine the role of two well-established objective rhinometric methods in the assessment of patients with CRS with nasal obstruction, comparing them with rigid nasal endoscopy and a subjective QoL instrument (SNOT-22 test). AR and NIPF showed to correlate partially, suggesting that they provide different and complementary information on nasal pathology and response to treatment. NIPF was proven to be an easy to perform and reproducible method, measuring the outcome of conservative treatment, especially in patients with nasal obstruction as the prominent symptom, as it could predict the subjective evaluation of response to medical treatment. As far as AR is concerned, it is a more specialized measurement of nasal patency which could be used before and at the end of medical treatment to confirm the resulted effect on nasal patency.

\section{Conflicts of Interest}

None to report

\section{References}

1. Fokkens W, Lund VJ, Mullol J, et al. European position paper on rhinosinusitis and nasal polyps. Rhinology. 2012; Suppl. 23: 88-110.

2. Ashan G, Drettner B, Ronge HE. A new tech- nique for measuring nasal airflow resistance to breathing, illustrated by the effect of histamine and physical effort. Ann Acad Regiae Sci. 1958; 2: 111-126.

3. Clement PAR. Committee report on stand- ardization of rhinomanometry. Rhinology. 1984; 22: 151-155.

4. Scadding G, Hellings P, Alobid I, et al. Diagnostic tools in Rhinology. EAACI position paper. Clin Translat Allergy. 2011; 1: 1-6. 
5. Youlten LJ. The peak nasal inspiratory flow meter: a new instrument for the assessment of the response to immunotherapy in seasonal allergic rhinitis. Allergol Immunopathol. 1980; 8: 344.

6. Nathan RA, Eccles R, Howarth PH, Steinsvag SK, Togias A. Objective monitoring of nasal patency and nasal physiology in rhinitis. Allergy Clin Immunol. 2005; 115: 442-459.

7. Holmstrom M, Scadding GK, Lund VJ, et al. Assessment of nasal obstruction. A comparison between rhinomanometry and nasal inspiratory peak flow. Rhinology. 1990; 28: 191-196.

8. Jones AS, Viani L, Phillips DE, et al. The objective assessment of nasal patency. Clin Otolaryngol. 1991; 16: 206-211.

9. Starling-Schwanz R, Peake HL, Salome CM, et al. Repeatability of peak nasal inspiratory flow measurements and utility for assessing the severity of rhinitis. Allergy. 2005; 60 795-800.

10. Wihl JA, Malm L. Rhinomanometry and nasal peak expiratory and inspiratory flow rate. Ann Allergy. 1998; 61: 50-55.

11. Phagoo SB, Watsoti RA, Pride NB. Use of nasal peak flow to assess nasal patency. Allergy. 1997; 52: 901-908.

12. Elbrond $\mathrm{O}$, Hilberg $\mathrm{O}$, Felding JU. Acoustic Rhinometry: a new method to evaluate the geometry of the nasal cavity and the epipharynx. Am J Rhinol. 1991; 5: 7-9.

13. Clement PAR, Gordts F. Consensus report on acoustic rhinometry and rhinomanometry Rhinology. 2005; 43: 169-179.

14. Numminen J, Ahtinen M, Huhtala $H$, et al Comparison of rhinometric measurements methods in intranasal pathology. Rhinology. 2003; 41: 65-68.

15. Kjærgaard T, Cvancarova M, Steinsvag KS Does Nasal Obstruction Mean That the Nose Is Obstructed? Laryngoscope. 2008 118: 1476-1481.

16. Hopkins C, Gillett S, Slack R, et al. Psychometric validity of the 22-item Sinonasal Outcome Test. Clin Otolaryngol. 2009; 34: 447-454.

17. Lane AP, Pine HS, Pillsbury HC. Allergy testing and immunotherapy in an academic otolaryngology practice: a 20-year review. Otolaryngol Head Neck Surg. 2001; 124: 9-15.

18. Lund VJ, Kennedy DW. Quantification for staging sinusitis. The staging and therapy group. Ann Otol Rhinol Laryngol. Suppl.1995; 167: 17-21.

19. Lim M, Lew-Gor S, Darby Y, Brookes N, Scadding G, Lund VJ. The relationship between subjective assessment instruments in chronic rhinosinusitis. Rhinology. 2007; 45: 144-147.

20. Ottaviano G, Scadding GK, Coles S, Lund VJ. Peak nasal inspiratory flow; normal range in adult population. Rhinology. 2006; 44: 32-35.

21. Timperley D, Srubisky A, Stow $N$, et al. Minimal clinically important differences in nasal peak inspiratory flow. Rhinology. 2011; 1: 37-40.

22. Hilberg O, Pedersen OF. Acoustic rhinometry: Recommendations for technical specifications and standard operating procedures. Rhinology. Suppl. 2000; 16: 3-17.

23. Fairley JW, Durham LH, Ell SR. Correlation of subjective sensation of nasal patency with nasal inspiratory peak flow rate. Clin Otolaryngol. 1993; 18: 19-22.

24. Jose J, Ell SR. The association of subjective nasal patency with peak inspiratory nasal flow in a large healthy population. Clin Otolaryngol. 2003; 28: 352-354.

25. Grymer LF, Pedersen, Hilberg O, et al. Acoustic rhinometry: evaluation of the nasal cavity with septal deviations, before and after septoplasty. Laryngoscope. 1989; 99: 1180-1187.

26. Marais J, Murray JA, Marshall I, et al. Minimal cross-sectional areas, nasal peak flow and patients' satisfaction in septoplasty and inferior turbinectomy. Rhinology. 1994; 32: 145-147.

27. Shemen L, Hamburg R. Preoperative and postoperative nasal septal surgery assessment with acoustic rhinometry. Otolaryngol Head Neck Surg. 1997; 117: 338-342.

28. Naito K, Miyata S, Saito S, et al. Comparison of perceptional nasal obstruction with rhi- nomanometric and acoustic rhinometric assessment. Eur Arch Otorhinolaryngol. 2001; 258: 505-508

29. Holmstrom M. The use of objective meas ures in selecting patients for septal surgery. Rhinology. 2010; 48: 387-393.

30. Larsson C, Millqvist E, Bende M. Relationship between subjective nasal stuffiness and nasal patency measured by acoustic rhinometry. Am J Rhinol. 2001; 15: 403-405.

31. Gilain I, Coste A, Ricolfi F, et al. Nasal cavity geometry measured by acoustic rhinometry and computed tomography. Arch Otolaryngol Head Neck Surg. 1997; 123: 401-405.

32. Corey JP, Nalbone VP, Ng BA. Anatomic correlates of acoustic rhinometry as measured by rigid nasal endoscopy. Otolaryngol Head Neck Surg. 1999; 121: 572-576.

33. Andre GJ, Vuyk RF, Ahmed HD, et al. Correlation between subjective and objective evaluation of the nasal airway. A systematic review of the highest level of evidence. Clin Otolaryngol. 2009; 34: 518-525.

34. Dávila I, Rondón C, Navarro A, et al. Aeroallergen sensitization influences quali ty of life and comorbidities in patients with nasal polyposis. Am J Rhinol Allergy 2012 26: 126-131.

35. Yamagiwa M. Acoustic evaluation of the efficacy of medical therapy for allergic nasal obstruction. Eur Arch Otorhinolaryngol. 1997; 254: 82-84.

Efklidis Proimos

Georgiladon 25

73133 Chania

Crete Greece

mob. +306944557577

e-mail: efklidispr@hotmail.com 